\title{
The rehabilitation treatment of pain syndrome in the chronic phase of spinal cord injuries
}

\author{
Florina Ojoga ${ }^{1,2}$, Brindusa Ilinca Mitoiu ${ }^{1,2}$, Delia Maria Clantau ${ }^{1,2}$ \\ ${ }^{1}$ Department of Medical Rehabilitation, "Carol Davila” University of Medicine and Pharmacy, \\ Bucharest, Romania \\ ${ }^{2}$ Medical Rehabilitation Clinic, INRMFB, 3rd Clinic, Bucharest, Romania
}

\begin{abstract}
Hipothesis. Spinal cord injuries represent a complex pathology, very frequent in the rehabilitation units, because in the last years there was an increase in the proportion of vertebral traumas, especially cervical ones, with more cases of tetraplegic patients admitted in the hospitals.

Purpose. The importance of the rehabilitation programme in the painful syndrome of the chronic phases is special, because aproximately $80 \%$ of the spinal cord injury patients have chronic pain, one third being severe, this interfering with the activities of daily living and affecting the quality of life.

Results and methods. There are many types of pain syndromes in patients with spinal cord injury, such as musculo-skeletal pain (nociceptive pain), visceral pain and neuropathic pain, all of them having a benefit from the rehabilitation programme and its specific treatment methods (physiotherapy, termotherapy, massage and kinetotherapy), but also from the pharmacologycal analgesic treatment, depending upon the severity of pain. Discussions. The purpose of the rehabilitation treatment after spinal cord injury includes maximizing physical and functional independence and prevention of the multiple secondary complications. The rehabilitation team is led by a rehabilitation physician, but includes other important members such as the patient, family members, physical therapists, psychologists and social workers, and all of these members must work together to eliminate the barriers to health and ensure the full participation of the patient in all aspects of community life.
\end{abstract}

Keywords: spinal cord injury, pain syndromes, rehabilitation

\section{HYPOTHESIS}

Spinal cord injuries are potentially letal events and their medical and non-medical effects can be devastating if they are not treated correctly, adequatelly and simultaneously. Spinal cord injuries determine physiological effects with a general character and multi-systemic functional generalised effects, that depend upon the reflex activity of the spinal cord distal to the lession. There are many perturbations of the reflex activity level of the spine during the lifetime of the patient, concerning the functionning of multiple systems of the body. Any dysfunction is the source of multiple disabilities and a source of secondary complication during the evolution of the disease $(1,9,19)$.
The annual incidence of spinal cord injuries varies for different countries, but usually doesn't overcome 20 cases per million per year, but in the last years there was an increase of these cases, with many tetraplegic patients being admitted in the hospital. The incidence is $63 \%$ for caucasians, $23 \%$ for africans, $12 \%$ for hispanics and less than $2 \%$ for the assian population. The spinal cord injuries are frequent in the male population the ratio between man and women being $4: 1$. The incidence is highest for the teenagers and young people less than 20 years, and then there is a peak for the persons over 60 years. The mean age of producing the event is 40 years old $(4,7)$.

The causes of spinal cord lesions are multiple: car or motor accidents (42\%), falls (27\%), violence 
(15\%) and sport accidents (8\%). Other non-traumautic causes are vascular diseases, tumors, inflammatory diseases, infections, demielinisant diseases, disc hernia, iatrogenic lesions, vertebral fractures secondary to osteoporosis, B12 vitamin deficiency and congenital diseases $(8,14,26)$.

\section{PURPOSE}

According to the latest studies, tetraplegia is more common $(50 \%)$ than paraplegia $(44 \%)$ and the life expectancy has increased in the last decades, the mortality rate being highest during the first year after injury $(6 \%)$ and declines significantly after that period $(7,8,10)$.

The functional outcome factors include, but are not limited to, preexisting medical conditions, concomitant injuries, secondary complications, cognitive impairments, age, body habitus, psychological factors, social factors and cultural factors $(9,18)$.

The rehabilitation goals after spinal cord injury include maximizing physical independence, becoming independent in direction of care and preventing secondary complications, so an interdisciplinary team approach is the model that is the best in the rehabilitation treatment of persons with spinal cord injury. The team is led by a rehabilitation physician and the other members are the patient, family members, physical therapists, occupational therapists, nurses, psychologists, recreational and vocational therapists and social workers. The person with spinal cord injury and the family members are essential members of the team, because if the patient does not participate in the rehabilitation program, it is not likely to be of much benefit. The rehabilitation team provides education on prevention and treatment of secondary complications (pulmonary, vasculary, cardiovasculary and autonomic system, on calcium metabolism and osteoporosis, gastrointestinal system, genito-urinary system and tegumentary system, such pressure ulcers), as well as training in bowel and bladder management. Psychologists try to reduce depression and anxiety, by supporting the patient and his family through the entire post-traumatic event using individual psychotherapy, cognitive and behavioral methods to provide coping with the event and group psychotherapy for additional support and information sharing. Physical and occupational therapists train persons with spinal cord injury in mobility, self-care skills and other activities of daily living. The purpose is to achieve adequate joint range of motion and strength through range of motion exer- cises, use of appropriate orthoses and resistance exercises $(10,19,22,28)$.

\section{RESULTS AND METHODS}

Spinal cord injury is a devastating event with a profound effect on the patient and family members and there are different factors (personal, interpersonal and cultural) that determine the successfully adjustment to disability, that is achieved when the disability is no longer the dominant concern in the person's life. This adjustment is individually variable, being estimated to occur after 2 to 5 years. A number of psychologic problems, primarily depression and anxiety can interfere with adjustment and quality of life and these problems lead to substance abuse, divorce, dependency, self-neglect and even suicide $(1,9,11,25)$.

Quality of life is difficult to define but we can describe it as a determination of the individual's satisfaction with life. In comparison with normal individuals, patients with spinal cord injury have a decreased well-being and a poorer state of health and a score lower on physical, emotional and social health domains of life. There is a relationship between the neurologic level and the subjective quality of life, because there are some positive factors such as mobility and activities of daily living independence, emotional support, good overall health, self-esteem, absence of depression, physical and social activities and integration, being married and employed, being more educated and living at home and also some negative factors which are more related to social disadvantages than to physical limitations $(9,16,21)$.

Approximately $80 \%$ of patients with spinal cord injuries have chronic pain, while approximately one third report chronic, severe pain that interferes with activities and affects the quality of life $(2,5)$.

There are many types of pain experienced by the patients with spinal cord injury, which have different etiologies and treatments and have been classified in different types. The most used classification organizes the types of pain into nociceptive and neuropathic categories. Nociceptive pain is defined as pain arising from activation of peripheral nerve endings or sensory receptors that are capable of transporting noxious stimuli. Neuropathic pain is defined as pain that arises as a direct consequence of a lesion or disease affecting the somatosensory system $(3,4,12)$.

Musculoskeletal pain (nociceptive pain) refers to pain occurring in a region where there are nociceptors within musculoskeletal structures (muscles, 
tendons, ligaments, joints, bones), at any location where there are musculoskeletal structures, including areas below the neurologic level of injury (17).

Because the patients with spinal cord injuries have bigger demands on the upper limbs during their daily activities and work, they have a higher incidence of overuse injuries and musculoskeletal pain. The overuse injuries are caused by repetitive motions and recurrent microtrauma and the treatment depends on the specific etiology, but in general there are some overlapping phases: control of inflammation and pain with protection, rest, ice application, compression, elevation and nonsteroidal anti-inflammatory drugs, then mobilization to regain joint range of motion, strengthening exercises when painless range of motion is achieved and functional restoration. The goal of this treatment is to achieve the previous level of function and prevention of the recurrences (19).

Shoulder pain is the most common and incapacitating upper limb overuse injury and can be caused by bicipital tendonitis, rotator cuff impingement syndrome, subacromial bursitis, capsulitis and osteoarthritis, its prevalence ranging from $40 \%$ to $50 \%$ in studies, in persons with chronic paraplegia or tetraplegia. The etiology of shoulder pain in patients with chronic tetraplegia includes pain that arises from shoulder instability, resulting from weakness of the muscles that stabilize the shoulder joint, as well as capsulitis and contracture caused by a lack of passive or active range of motion and spasticity (6).

Specific treatment and prevention from shoulder pain from the rotator cuff in patients with spinal cord injury include strengthening, stretching, postural exercises and avoidance of activities that cause the impingement. We must strengthen the dynamic shoulder stabilizers in a balanced fashion, especially the posterior shoulder muscles, including the external rotators, the posterior scapular muscles (rhomboid and trapezius) and the adductors. Use of wheel-chairs realizes strengthening of the antagonists to these muscles (the anterior shoulder muscles), so the stretching of these is also necessary to achieve a balanced shoulder, because these muscles often are hypertrophied and contracted during constant use of wheel-chair propulsion and transfer activities. Elbow pain in paraplegic patients is also common ( $16 \%$ to $35 \%)$, often caused by lateral epicondylitis $(22,28)$.

Visceral (nociceptive) pain is usually located in the thorax, abdomen or pelvis, primarily generated in visceral structures. This category includes abdominal pain caused by fecal impaction, bowel ob- struction, bowel perforation, cholecystitis, pancreatitis, appendicitis, splenic rupture, bladder perforation, pyelonephritis. The pain is characteristically vague and poorly localized, there is tenderness of the visceral structures on palpation of the abdomen. Visceral pain is associated with symptoms of autonomic dysfunction, anorexia, altered bowel patterns such as constipation, nausea or vomiting (29).

Autonomic dysfunction headache pain can be severe and is usually described as pounding, most common in patients with a neurologic level of injury at or above T6. It is associated with elevated blood pressure, diaphoresis, piloerection, cutaneous vasodilatation above the level of injury, bradycardia or tachycardia, nasal stuffiness, conjunctival congestion and mydriasis. The syndrome is usually triggered by a noxious stimulus inferior to the level of injury such as bowel impaction or urinary tract infection (15).

Neuropathic pain can appear at the level of spinal cord injury or below it (26).

Neuropathic pain at the level of spinal cord injury means neuropathic pain perceived in a segmental pattern within the dermatome of the neurologic injury and/or within the three dermatomes below this level and not in any lower dermatomes. The pain can be unilateral or bilateral and is suggested by allodynia or hyperalgesia and by the following descriptions: hot-burning, tingling, sharp, shooting, squeezing, painful cold, electric-shock like and numb. It is often difficult to distinguish between the two subcategories of at-level spinal cord injury pain, spinal cord pain and radicular pain, because both are involved in a traumatic spinal cord injury. Radicular pain is in generally unilateral and radiating in a dermatomal pattern. Atlevel radicular pain is usually paroxysmal, whereas at-level spinal cord pain is typically constant. Syringomyelia often presents initially with at-level spinal cord pain, either on coughing or spontaneously. The character of pain in syringomyelia is burning or dull aching, but it can be also sharp, electrical or stabbing, localized unilaterally or bilaterally (18).

Neuropathic pain below-level of spinal cord injury refers to neuropathic pain perceived more than three dermatomes below the neurologic level of injury. The presence of this type of pain is suggested by the same pain descriptors mentioned above. Allodynia or hyperalgesia can be present and the distribution is generally not dermatomal but regional, enveloping large areas such as the anal region, the bladder, the genitals, the legs, or commonly the en- 
tire body below the neurologic level of injury. It is frequently continuous, although the intensity of pain can fluctuate from many factors such as psychologic stress, anxiety, fatigue, smoking, noxious stimuli below the level of injury and weather changes.

Treatment of neuropathic pain (at or below the level of spinal cord injury) is not very effective, but there are some oral pharmacologic agents, such as pregabalin, gabapentin, tramadol and amitriptyline with some effectiveness in some randomized controlled trials for the treatment of neuropathic pain after spinal cord injuries. Also, there are also some other medications commonly prescribed, including the selective serotonin and norepinephrine reuptake inhibitors, opioids (oral, transdermal, intratechal) and neurotoxins (intra-techal ziconotide) which relieve the painful neuropathic syndrome. Modalities including desensitization techniques for evoked pain, massage and especially exercise can be beneficial. Psychologic interventions including education, cognitive-behavioral therapy, relaxation and hypnosis can allow these patients to manage the pain and to minimize its impact on their quality of life. Neuro-interventional techniques such as surgical nerve root decompression and transforaminal epidural steroid injections for radicular pain, and dorsal root entry zone micro-coagulation for spinal cord pain $(12,26)$.

Other neuropathic pain is represented by compressive neuropathy that occurs in a peripheral nerve distribution distal to the root level. Symptoms include either spontaneous or evoked numbness or tingling in a specific peripheral nerve distribution. This includes median, ulnar, radial and axillary neuropathies in paraplegic patients. The signs and symptoms of the carpal tunnel syndrome, represented by numbness or tingling of the thumb, index or middle fingers, abnormal sensation on testing, or numbness and tingling with provocative tests such as Tinel or Phalen signs, are common in persons with paraplegia. The recorded prevalence correlated with the time since injury is $42 \%$ for a group 0 to 5 years postinjury, $74 \%$ for 10 to 14 years postinjury and $86 \%$ for 20 or more years postinjury, so the prevalence increases with the time elapsed from the spinal cord injury. This syndrome results from a combination of repetitive trauma during propulsion of manual wheel-chairs, and ischemia from repetitive marked increases in carpal canal pressures during transfers from one seating surface to another. A higher risk for developing this type of pain are those over-weighted pa- tients or the ones that use improper wheel-chair propulsion $(13,23)$.

The treatment and prevention strategies for the compressive neuropathies occurring at the wrist include avoidance of weight-bearing on a flexed or extended wrist, weight loss, using of the lightest possible wheelchair that permits the needs of the patient, use of power and power-assist wheel-chairs and instruction in training in an efficient wheelchair propulsion model. This is a model that minimizes the forcefulness and frequency with which the wrist strikes the wheel (long and smooth arm strokes). Side-to-side or forward lean pressure reliefs should be substituted for push-up pressure reliefs (24).

\section{DISCUSSIONS}

Spinal cord injuries are devastating events that result in physical disability and impaired function of various organs and systems and unfortunately, despite a long time of research, a curative treatment doesn't exist, but a great progress has occurred in the management of its associated conditions. This is reflecting in an increase of the life expectancy of these patients and reducing in morbidity, reflected in reduced hospital time of stay and fewer hospitalizations during follow-up years. Application of modern technology has enhanced the function and quality of life of many persons with spinal cord injury. Current wheel-chair designs and seating systems are superior to older models and permit increased mobility and allow safe sitting for much longer periods of time and people with disability use also computers in their daily lives for educational and vocational purposes (27).

In the rehabilitation process, pain should be examined every day by the rehabilitation team. Usually the patient rates pain using the visual analogue scale (VAS), a scale from 0 to 10 , where 0 is no pain and 10 is severe, disabling pain. There are also some other scales, as The International Spinal Cord Injury Basic Pain Data Set in which the patient selfreport measure is used, using a series of questions related to the impact of pain on various aspects of daily activities and life satisfaction, location, intensity and duration of pain. The Wheelchair Users Shoulder Pain Index measures the impact of shoulder pain on transfers, self-care, wheel-chair mobility and general activities, using a scale from 0 to 10. Total score ranges from 0 to 150 , higher scores indicating a greater impact of pain on the wheelchair mobility $(1,2,6)$. 
But the health and function of patients with spinal cord injury are still at risk without proper medical and nursing care, social support, appropriate equipment, supplies and medications. They continue to experience problems of the urinary and bowel dysfunction, many have chronic pain that interferes with their quality of life, so we must increase our understanding of the value of physical exercise and proper diet to reduce the high prevalence of obesity, diabetes and cardiovascular disease in patients with spinal cord injury (27).

The functional outcome after spinal cord injuries is dependent, primary, for complete injuries, on the level of motor function (in lower-level lesions there are more intact muscles) and there is greater potential for independence in mobility tasks and activities of daily living $(1,2,9)$.

Goals in the functional rehabilitation treatment should reflect what is important for the patient, because this will increase motivation and enhance patient autonomy. Long-term goals should focus on activity and social participation, not body structure and functional impairments and these goals should be specific in what the patient will achieve $(9,23)$.
The ultimate goal of persons with spinal cord injury and those who care for them is to find a proper resolution for their condition, which is to reverse the neurologic damage of the spinal cord, but until this far-away goal is reached, the patients, their families, their caregivers and the society must work together to eliminate barriers to health care and ensure their full participation in all the aspects of the community life, so their quality of life to be maximized $(1,9,10)$.

\section{CONCLUSIONS}

Spinal cord injuries have a higher incidence in the latest years, with higher costs, and result in dramatic changes in the individual's life. These changes can leas to altered and limited mobility, self-care and ability to participate in the daily living activities.

Rehabilitation is an important element for achieving an active life and the rehabilitation team plays a key role in that process.

\section{REFERENCES}

1. Bickenbach J, Biering-Sorensen F, Knott J et al. A global picture of spinal cord injury. In Bickenbach J, Officer A, Shakespeare T et al, editors: International perspectives of spinal cord injury, Geneva, 2013, World Health Organization Press.

2. Bryce TN. Pain management in persons with spinal cord injury. In Lin VW, editor: Spinal cord medicine: principles and practice, New York, 2010, Demos.

3. Bryce TN, Biering-Sorensen F, Finnerup NB et al. International Spinal Cord Injury Pain Classification: part I. Background and description. Spinal Cord 50:413-417, 2012.

4. Cardenas DD, Bryce TN, Shem K et al. Gender and minority differences in the pain experience of people with spinal cord and injury. Arch Phys Med Rehabil 85:1774-1781, 2004.

5. Chiodo A. Pain management with interventional spine therapy in patients with spinal cord injury: A case series. J Spinal Cord Med 28:338-342, 2005.

6. Curtis KA, Tyner TM, Zachary L et al. Effect of a standard exercise protocol on shoulder pain in long-term wheelchair users. Spinal Cord 37:421-429, 1999.

7. DeVivo MJ. Epidemiology of traumatic spinal cord injury. In Kirshblum S, Campagnolo DI, DeLisa JA, editors: Spinal cord medicine, Philadelphia, 2002, Lippincott Williams \& Wilkins, pp69-81.

8. DeVivo MJ. Epidemiology of traumatic spinal cord injury: Trends and future implications. Spinal Cord 50:365-372, 2012.

9. Dijkers MP. Quality of life of individuals with spinal cord injury: A review of conceptualization measurement, and research findings. J Rehabil Res Dev 42:87-110, 2005.

10. Donovan WH. Donald Munro lecture. Spinal cord injury-past, present and future. J Spinal Cord Med 30:85-100,2007.

11. Fann JR, Bombardier CH, Richards JS et al. Depression after spinal cord injury: Comorbidities, mental health service use, and adequacy of treatment. Arch Phys Med Rehabil 92:352-360, 2011.

12. Finnerup NB, Baastrup C: Spinal cord injury pain: mechanisms and management. Curr Pain Headache Rep 16:207-216, 2012.

13. Gellman H, Chandler DR, Petrasek J et al. Carpal tunnel syndrome in paraplegic patients. J Bone Joint Surge AM 70:517-519, 1988.

14. Kamin SS. Vascular, nutritional and other diseases of the spinal cord. In Kirshblum S, Campagnolo DI, DeLisa JA, editors: Spinal cord medicine, Philadelphia, 2002, Lippincott Williams \& Wilkins, pp 512-526.

15. Krassioukov A, Warburton DE, Teasell $R$ et al. A systematic review of the management of autonomic dysreflexia after spinal cord injury. Arch Phys Med Rehabil 90:682-695, 2009.

16. Krause JS, Kewman D, DeVivo MJ et al. Employment after spinal cord injury: An analysis of cases from the model spinal cord injury systems. Arch Phys Med Rehabil 80:1492-1500, 1999.

17. Little JW, Burns SP. Neuromusculoskeletal complications of spinal cord injury. In Kirshblum S, Campagnolo SI, DeLisa JA, editors: Spinal cord medicine, Philadelphia, 2002, Lippincot Williams \& Wilkins, pp 241-252.

18. McKinley WO. Nontraumatic spinal cord injury: Etiology, incidence and outcome. In Kirshblum S, Campagnolo SI, DeLisa JA, editors: Spinal cord medicine, Philadelphia, 2002, Lippincot Williams \& Wilkins, pp 471-479.

19. National Spinal Cord Injury Statistical Center: 2012 Annual statistical report for the spinal cord injury model systems-complete public version. Birmingham, 2013, University of Alabama at Birmingham.

20. Schmitz TJ. Traumatic spinal cord injury. In O'Sullivan SB, Schmitz TJ, editors: Physical Rehabilitation: assessment and treatment, Philadelphia 2011, FA Davis.

21. Selvarajah S, Hammonsd E, Haider A et al. The burden of acute traumatic spinal cord injury among adults in the United States: An update. J Neurotrauma 31:228-238, 2014. 
22. Van Middendorp JJ, Hosman AJ, Donders AR et al. A clinical prediction rule for ambulation outcomes after traumatic spinal cord injury: A longitudinal cohort study. Lancet 377:1004-1010, 2011.

23. Whiteneck $G$, Adler $C$, Biddle AK et al. Outcomes following traumatic spinal cord injury :clinical practice guidelines for health-care professionals. Washington, 1999, Paralyzed Veterans of America.

24. Marino RJ, Burns S, Graves DE et al. Upper-and-lower-extremity motor recovery after traumatic cervical spinal cord injury: An update from the National Spinal Cord Injury Database. Arch Phys Med Reahabil 92:369-371, 2011.

25. Hoffman JM, Bombardier CH, Graves DE et al. A longitudinal study of depression from 1 to 5 years after spinal cord injury. Arch Phys Med Rehabil, 92:411-418, 2011.
26. Kirshblum SC, Waring W, Biering-Sorensen F et al. Reference for the 2011 Revision of the International Standards for Neurological Classification of Spinal Cord Injury. J Spinal Cord Med 34:547-554, 2011.

27. Scivoletto G., Morganti B, Ditunno $P$ et al. Effects on age on spinal cord lesions patients' rehabilitation. Spinal Cord 41:457-464, 2003.

28. Wilson JR, Forgione N, Fehlings MG. Emerging therapies for acute traumatic spinal cord injury. CMAJ 185:485-492, 2013.

29. Ditunno IF, Little JW, Tessler A et al. Spinal shock revisited: A four-phase model. Spinal Cord 42:383-395, 2004. 